\title{
Gene expression analyses of palmitate-induced steatosis in human hepatocytes
}

\author{
K. J. Levy ${ }^{1}$, D. J. Mazzatti ${ }^{2}$ and J. B. Moore ${ }^{1}$ \\ ${ }^{1}$ Faculty of Health \& Medical Sciences, University of Surrey, Guildford GU2 7XH, UK and ${ }^{2}$ Unilever Corporate Research, \\ Sharnbrook MK44 $1 L Q, U K$
}

Non-alcoholic fatty liver disease (NAFLD) is the emergent hepatic consequence of obesity and insulin resistance with an estimated prevalence in developed countries of between $20 \%$ and $30 \%$. Clinically, NAFLD may progress from hepatic steatosis to the more severe non-alcoholic steatohepatitis (NASH), which can lead to fibrosis, cirrhosis and end-stage liver disease. While NAFLD pathogenesis is likely to be influenced by multiple genetic and environmental factors, converging data suggest that SFA play a key role in the development and progression of $\mathrm{NASH}^{(1)}$.

The objective of these experiments was to identify changes in gene expression in an in vitro model of progressive steatosis. Human hepatocytes ( $\mathrm{HuH} 7$ cells) were treated with either vehicle or an increasing concentration of palmitate $(50,100$ or $200 \mu \mathrm{M}) \mathrm{for} 24 \mathrm{~h}$ and total RNA was isolated. An initial 'candidate gene' approach was taken and quantitative PCR primers were designed for potential housekeeping genes ( $\beta$-actin (ACT) and glyceraldehyde 3-phosphate dehydrogenase (GAPDH)) and several genes involved in lipid metabolism and inflammation that have been implicated in NAFLD (LDL receptor (LDLR), SREBF1, PPAR $\alpha$, PPAR $\delta$, PPAR $\gamma$ and transforming growth factor $\beta 1$ (TGF $\beta 1)$ ). Data were analysed by one-way ANOVA and Dunnett's multiple comparison when appropriate.

There were no differences found in the levels of ACT and GAPDH mRNA between groups $(P=0.0789$ and $P=0.1942$ respectively), but since ACT showed a potential trend for modulation by palmitate levels, GAPDH was used for normalization. Only LDLR mRNA levels were altered significantly with palmitate treatment $(P=0.0198)$ and were induced fourfold with $200 \mu \mathrm{M}$-palmitate (four times the physiological dose). No differences were seen in expression levels of SREBF1 $(P=0.2982), \operatorname{PPAR} \alpha(P=0.4093)$, PPAR $\delta(P=0.8474)$, $\operatorname{PPAR} \gamma(P=0.4063)$ and TGF $\beta 1(P=0.1123)$.

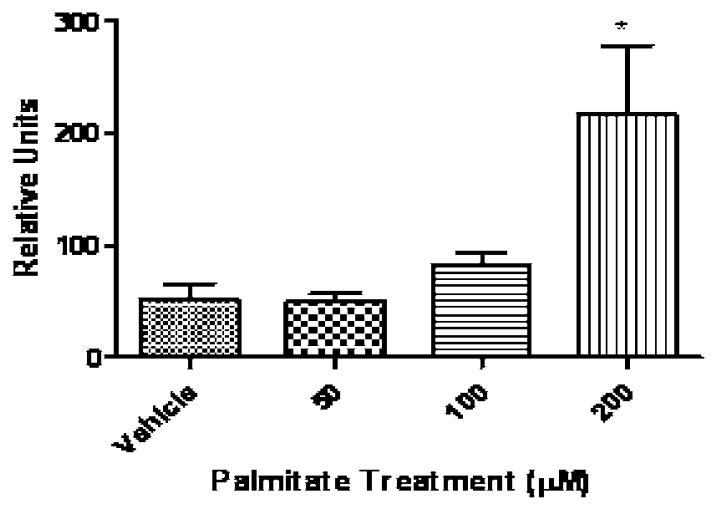

Figure. LDLR mRNA expression. HuH7 cells were cultured with vehicle or palmitic acid for $24 \mathrm{~h}$ and LDLR mRNA levels were measured by quantitative PCR and normalised to GAPDH levels. Values are means with their standard errors represented by vertical bars for three experiments. Mean value was significantly different from that for the vehicle (one-way ANOVA and Dunnett's multiple comparison): * $P=0.0198$.

These data prompted the undertaking of an unbiased approach to analysing gene expression using Agilent ${ }^{\mathrm{TM}}$ whole-genome microarrays $^{\mathrm{m}}$ (Agilent Technologies UK Ltd, Stockport, Ches., UK).

Data from independent experiments (n 3, twelve microarrays) were analysed for differences in gene expression between vehicle-, 50, 100 or $200 \mu \mathrm{M}$-palmitate-treated cells using GeneSpring software (GX 7.3.1; Agilent Technologies UK Ltd) and a dose-dependent effect was exhibited at palmitate concentrations of 100 and $200 \mu \mathrm{M}$. Probes found commonly differentially expressed (1069) were subjected to Ingenuity ${ }^{\mathrm{TM}}$ Pathway Analysis (Ingenuity Systems Inc., Redwood City, CA, USA), which identified several functional networks and signalling pathways altered in palmitate-induced steatosis. Underscoring the relevance of the in vitro model, the most significantly dysregulated network identified was associated with lipid metabolism, PPAR signalling $(P=0.0008)$ was the most significantly affected canonical pathway and PPAR $\alpha / \mathrm{RXR} \alpha$ activation was significantly down regulated $(P=0.004)$.

In conclusion, palmitate treatment induces specific changes in mRNA expression in human hepatocytes. Further analysis of altered networks in palmitate-induced steatosis may identify molecular mechanisms for the progression of NAFLD.

This project was funded by University of Surrey pump priming funds.

1. Preiss D \& Sattar N (2008) Clin Sci 115, 141-150. 